\title{
Research on the Supply Chain Problems and Optimization of Live Streaming E-commerce
}

\author{
Yue Wang ${ }^{1}$, Lina Fang1, * \\ ${ }^{1}$ School of Business Administration, Liaoning University of Science and Technology, Anshan, Liaoning, 114000, China. \\ * Corresponding author: Lina Fang (Email: 442754168@qq.com)
}

\begin{abstract}
The acceleration of the pace of mobile Internet construction and the outbreak of the COVID-19 epidemic have created new opportunities for the development of the live broadcasting economy. More and more businesses provide consumers with more visual and personalized services through live broadcasting. While developing the rapid development of live streaming e-commerce, it also puts forward new requirements for the whole supply chain. The optimization of the supply chain can comprehensively improve the operation efficiency of enterprises in all links. This article combines the meaning and basic structure of the live streaming e-commerce supply chain combined with the concept of live streaming e-commerce and supply chain. Compared with the traditional supply chain, the live streaming e-commerce supply chain has high information interaction efficiency, supply and demand matching efficiency and service efficiency. The article believes that the supply chain facing supply and undertaking ability, response speed and low coordination and sharing of supply chain should build reverse integrated supply chain; strengthen supply chain information sharing through digital technology, ensure production stability, improve the service quality of all links of the supply chain, and enhance competitiveness.
\end{abstract}

Keywords: Live streaming e-commerce, Supply chain, challenge, Optimization.

\section{Introduction}

In 2016, China live electricity began to sprout, first open the live mode is mushroom street, then jingdong taobao successively on the development of live sales, 2016-2017 is a period of rapid development, into 2018, head short video platform into the bureau, with the help of its considerable traffic and many anchors, obtained good effect with goods in the short term. In 2019, live streaming e-commerce formed a tripartite pattern of Tao, Kuaidi and Dou. With the outbreak of the epidemic in early 2020 , live streaming e-commerce has become one of the life-saving channels in various industries, and the development speed is booming beyond imagination. According to data from the Ministry of Commerce, as of November 2020, there were more than 20 million businesses making live broadcasts, and the growth rate of live streaming e-commerce is estimated to be $90 \%$ in 2021 . This high growth rate is expected to last for a long time. All live streaming platforms and content platforms have also launched technological upgrading achievements and new plans for enterprise operation, and live streaming e-commerce has entered the full flowering stage. It is internal logic that live streaming is so popular and has a large fan base. First, the platform can accurately analyze fan data, constantly guide and create demand, shift from passive consumption to active consumption, followed by group time fragmentation, short video becoming popular trend information explosion, web celebrity culture, crowd mentality, and its tendency to make fast and stable consumption decisions, increasing national disposable income, and higher consumer demand in experience efficiency. Although the rapid development of live streaming e-commerce, the supply chain problems behind live streaming e-commerce also face many challenges, which has become the core problem of various enterprises. There is still a lot of room for live streaming development and progress of e-commerce in the future.

\section{Construction of Live Streaming E- commerce Supply Chain}

\subsection{Meaning of Live Streaming E-commerce}

According to the market research report of China live streaming e-commerce market in the first half of 2020, live streaming e-commerce refers to the process of relying on anchors on live broadcasting platforms to show goods to consumers or provide services through live broadcast, with goods here including physical and virtual goods. The essence of live broadcasting e-commerce is a combination of live broadcasting and e-commerce. In the process of watching live broadcasting, consumers can fully understand the real-time interaction between the goods and the anchors, and also communicate consumption experience with other customers. which is a professional shopping guide service that cannot be provided by traditional e-commerce. Live streaming ecommerce is not simply conducting content production, but an Internet business model [1] that connects with users and commodities through live broadcast, thus directly generating transactions and bringing benefits to the main broadcast, merchants and users. Combined with the above research, this article summarizes the live streaming e-commerce business as, including the live streaming through the live streaming platform, connecting suppliers and consumers to conduct commodity transactions or provide services, collectively referred to as the live streaming e-commerce business. Compared with the traditional business model, the characteristics of real-time, interaction, universality and trust endorsement of live streaming e-commerce will bring consumers a high experience sense of [2].Live streaming ecommerce is "live streaming + e-commerce". At present, the live streaming e-commerce industry has two general operation modes. One is for e-commerce platforms to open live broadcast rooms to attract consumers. It can be intuitively summarized as the e-commerce + live broadcast mode. One 
is the live broadcast + e-commerce mode of the content platform introducing the third-party e-commerce platform.

\subsection{Supply Chain Concept}

"Supply chain" refers to the value chain connected to raw materials and parts suppliers, product manufacturing enterprises, circulation enterprises, retail enterprises and end users, and is the [3] of the whole process from the customer needs to providing the customer with the required products and services. Supply chain includes suppliers, manufacturers, distributors, retailers and consumers, they form the supply chain, through regulating and control resources related to the enterprise, affect the whole process from raw material procurement, to the consumer, seeking to minimize costs and maximize benefits, network supply chain will have a significant impact, but the consumer node is always the final node of the supply chain, determines the operation and development of other supply chain nodes. From a broad perspective, the supply chain is extensive in the process of our production and life, from our production to circulation to transaction to consumption. The process from putting into production to consumption is also a supply chain system. Defining a supply chain in a narrow perspective refers to the network chain structure[4] composed of all node enterprises in the whole process of the product or service provided to the end user in the process of production and circulation.

\subsection{Meaning and Basic Structure of Live Streaming E-commerce Supply Chain}

From the perspective of supply chain, the basic business process of live streaming e-commerce is composed of four parts: sales end, supply end, consumption end and platform end, which correspond to the four links [5] of the commercial value chain such as e-commerce anchors, suppliers, consumers and platforms. The upstream of the industrial chain of live broadcast e-commerce is product suppliers, such as manufacturers providing products, the midstream is content exporters, such as live broadcast institutions, media institutions and major social media and e-commerce platforms, and the downstream is consumer[6]. The supply chain of live streaming e-commerce consists of three links. One is the production link. The producer is the "producer" of the supply chain of live streaming e-commerce. It can attract more live streaming merchants to cooperate through the appropriate wholesale price or preferential price. Some manufacturers will directly become live streaming ecommerce cooperation with express delivery companies to reduce the complexity of links and improve sales volume. Second, the sales link, through the way of live broadcast to directly connect raw manufacturers and consumers, reduce the traditional dealers, dealers, retailers and other redundant links. This link is mainly for platform merchants to sell on the live broadcast platform through cooperation with manufacturers, and to provide consumers with corresponding products and services. After the completion of orders, they will cooperate with logistics companies to provide order information and deliver transportation products. The third is the logistics link. Logistics providers are the "transmitters" of the online shopping supply chain, which is an indispensable link between e-commerce and consumers. The service and transportation speed of logistics providers will also directly affect consumers' online shopping experience [7].

Based on the above research, it is found that the overall concept and essential nature of the live broadcasting ecommerce supply chain are not very different from the traditional supply chain. The live-streaming e-commerce supply chain is only an integrated supply chain that is more short, fast and efficient driven by the development of the live broadcasting economy, and has its own characteristics. So combined with the concept of supply chain and live electricity characteristics this study will live electricity supply chain as, overall live electricity supply chain service link is divided into production, circulation, consumption, basic business process consists of the supply, sales and consumer three parts, supply corresponding product processing suppliers, sales corresponding live platform, consumption corresponding end consumers, compared with the traditional supply chain from production to circulation link to the final consumption link reduced brands, dealers, agents, retailers. The comparison flow chart of live streaming e-commerce supply chain and traditional supply chain is shown in Figure 1:

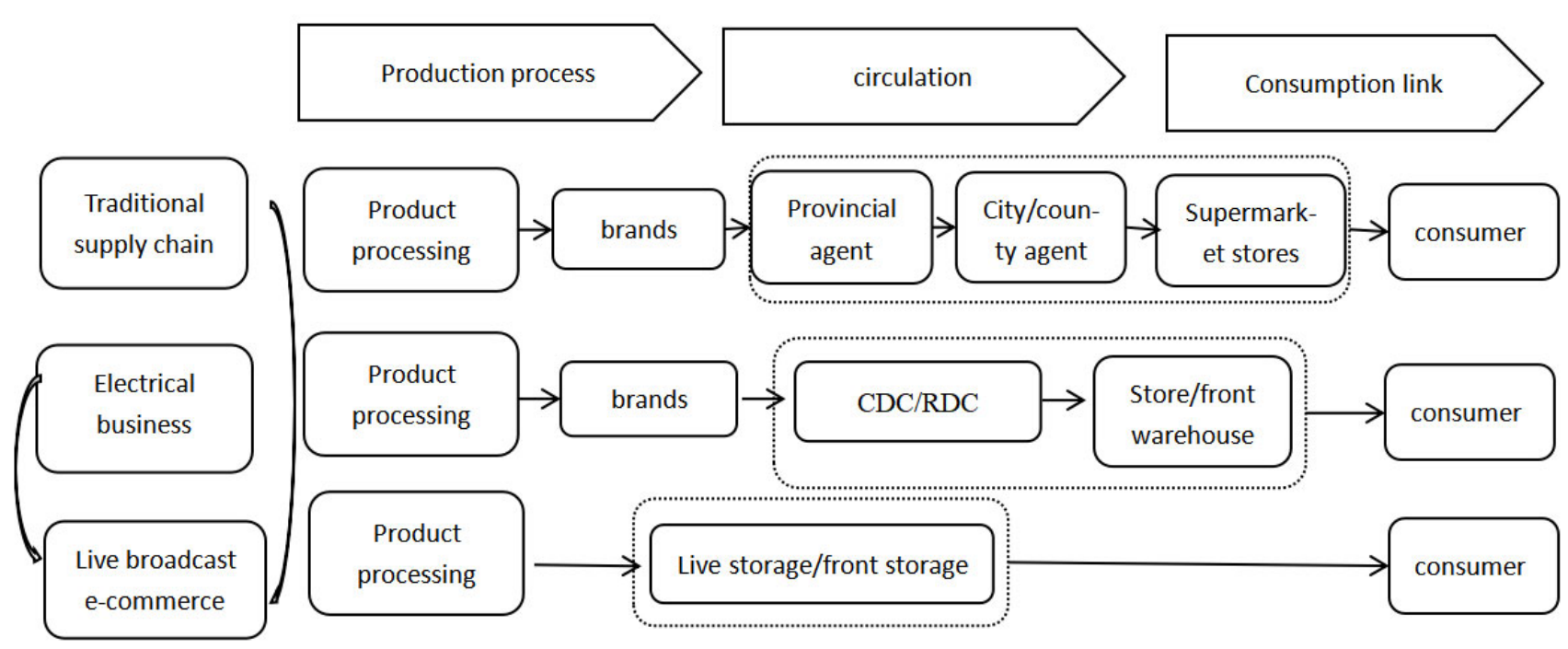

Figure 1. Supply chain process comparison diagram 


\section{Innovative Characteristics and Advantages of Live Streaming E- commerce Supply Chain}

Through the comparative analysis of the above and the traditional supply chain, it can be seen that compared with the traditional supply chain, the live streaming e-commerce supply chain has the following innovative characteristics:

First, the information interaction efficiency of live streaming e-commerce supply chain has been improved, with multi-dimensional product information dynamic display ability and "face to face" situation interaction ability [8]. Compared with single static information such as text and pictures of traditional e-commerce, live e-commerce can display dynamic information from multiple angles and at close range; consumers will have face-to-face situations. The live streaming platform provides real-time communication platform, allowing consumers to talk with the anchor, raise questions and needs to the live broadcast anytime and anywhere, and anchors will provide professional guide services for consumers. Consumers can not get the needs and questions in the broadcast room not only be answered by anchors, but also feedback from other consumers, consumers exchange commodity experience in the live broadcast to provide more relevant information for consumption decisions.

Second, the supply and demand matching efficiency of the supply chain of live streaming e-commerce is improved. Traditional e-commerce companies rely on consumers to actively search, and their consumer demand comes from advertising, marketing or life needs. They gather people with shopping purpose, and their products are waiting for passive consumption. Live streaming e-commerce is creating demand for consumers based on fans' trust in anchors and attractive advertising information. Anchors not only display rich commodity information but also provide relevant professional knowledge, consumers can also learn to wear and makeup to enhance interesting enjoyment. In the live broadcast, anchors have a deeper understanding of consumer consumption habits and product preferences, and collect feedback on consumers 'needs and preferences. Manufacturers will provide personalized and customized services according to the changes of consumer market demand and consumer needs, so as to improve consumers' sense of participation.

Third live electricity supply chain service efficiency improvement, some anchors will even move the studio into the factory, the anchor to the real production workshop as the background, full-round real-time product production process, product information visualization, not only close display the product color, material and other attributes and through the vivid description of inspection and trial experience. In this process, consumers will reduce a lot of search costs to obtain more effective commodity information, and consumers' intuitive perspective will generate trust and eliminate doubts. Live merchants as the core of connecting producers and consumers in the process of live understanding, follow up, feedback, solve consumer demand, and timely response to producers, producers according to the live business information of timely and efficient organization production complete orders, constantly reduce the middle link, inventory pressure is relatively small, improve cost performance, improve service efficiency.

\section{Challenges Facing the Live Streaming E-commerce Supply Chain}

\subsection{Demand Is Difficult to Predict the Supply and Undertaking Capacity Challenges}

The consumption end of the live streaming e-commerce supply chain is directly connected with consumers through the live broadcast platform. The economic model oriented by consumer demand is uncertain. The formation of explosive products is affected by multiple factors, and it is relatively difficult to predict. This situation is prone to at least two following problems. One is the demand Angle, out of different consumer consumption habits and personal characteristics, consumer-oriented personalized products are often complex and diverse, under this pressure, the supply of suppliers often can not meet the customer needs of some hot style goods, will lead to customer consumption experience decline, cause customers' dissatisfaction with the platform stores. Second, the supply perspective: affected by time and consumer preferences, online live broadcast with goods is often uncertain. If the relevant merchants blindly prepare the goods and arrange the shipping rules in advance under the needs of unknown customers, if the sales volume is good, they will meet the demand and achieve the effect of rapid distribution. However, if the live broadcast sales situation is not good, it will cause goods accumulation and supply link disorder and other problems. Therefore, how to predict and solve the problem of the unstable number of goods in the process of network live broadcast, and reasonably arrange the product supply chain behind them, is a major challenge faced by live broadcast with goods. After reasonably predicting the sales volume of goods before live broadcast delivery, it is important to improve the efficiency after generating orders, such as enhancing the process flexibility of goods stocking, logistics and distribution, which is an important parameter to solve the background supply chain problem of live broadcast.

\subsection{Reducing the Product Life Cycle Brings Response Speed Challenges}

Restricted by the speed of the Internet and the network platform application, the traditional e-commerce mode is relatively limited in terms of the depth or breadth of the impact. For example, when consumers buy goods, they can only watch text pictures and comments from the buyer's show, resulting in a low consumption impulse. In addition, with the long product cycle, slow iteration speed and other reasons, the traditional e-commerce has low requirements for the process speed and effectiveness of the supply chain. However, in the new situation of live streaming e-commerce, tremendous changes have occurred. In particular, the consumption mode has changed from the supply end to the consumption end, and enterprises and platform logistics have met the needs of consumers. As a result, in the process of live broadcasting with goods, we should focus on meeting the personalized needs of different consumers in different periods and angles. Based on this, in order to ensure their own attraction, the live broadcasting platforms will continuously update their product style and style to enhance customer stickiness, so as to improve their business sales and expand their fan layer. These characteristic demand models at the consumer end will force 
enterprise reform and logistics supply chain acceleration. For example, a web celebrity anchor may have to show several or even dozens of styles of related products at a first broadcast, and the amount of goods with a live broadcast may be the amount of months or even a year of offline entities. Therefore, how to deal with the requirements of accelerating logistics and upgrading of production enterprises brought by the continuous shortening of the production life cycle, how to grasp the development opportunities brought by them, make flexible, effective, fast and rapid innovation and solve the speed of the production process, and optimize the logistics distribution process are urgent problems to be solved by live streaming e-commerce.

\subsection{Low Degree of Coordination and Information Sharing in All Links of the Supply Chain Chain}

With the change of marketing focus oriented by customer personalized needs, consumers' consumer demand presents the characteristics of diversified, personalized and refined products. As the intermediate link of the live streaming ecommerce supply chain, the live broadcast platform and anchors cooperate with suppliers in the upstream of the supply chain to understand the products, obtain price discounts, and give timely feedback to customers' needs and opinions on the products, introduce the products to consumers in the downstream of the supply chain, and provide more visual and personalized services. Most live electricity and suppliers coordination is not close, disconnect, live electricity only agent sales level, not timely feedback customer demand and product opinions to suppliers, not comprehensive understanding of commodity information through suppliers simply provide commodity information, not detailed answer customer details, suppliers did not strengthen follow-up subsequent effect and problem processing, unable to get feedback to improve the quality of goods, for these problems, light sales decline, seriously lose customer trust, and even far from consumers, affect long-term development.

The explosive growth in orders illustrates both a surge in commodity sales and an unprecedented challenge to the logistics supply chain. However, the low price of the live streaming e-commerce supply chain is also reducing the service cost of logistics. The possibility of commodity damage or loss increases, while the transportation speed is also affected, and the delivery speed is slow down. For example, some products themselves are high-quality products, but in the distribution link of human reasons and time limitations, resulting in poor quality of goods, affect consumer satisfaction, and make consumers have greater opinions. Logistics and transportation speed and service problems are particularly prominent, which seriously restricts the development of logistics enterprises. As logistics enterprises are directly linked to live streaming e-commerce, the logistics service level will also directly affect the service level of live streaming e-commerce. How to balance these contradictions is also a big challenge.

\section{Supply Chain Optimization of Live Streaming E-commerce}

\subsection{Build A Reverse Integrated Supply Chain}

Behind the demand and consumption driven by live streaming e-commerce, producers' supply of products, order acceptance and effective management ability are the essence of network live streaming e-commerce. A series of difficult problems caused by the above situation are the bottleneck restricting the current rapid development of live streaming ecommerce. From the perspective of supply chain, live streaming e-commerce relies on the drainage role of the platform and anchors as the fulcrum, uses online organic interaction to understand and collect the diversified needs of consumers, and enhance customer stickiness through the strong visual impact of the live broadcast process. In this process, the use of network drainage, to organically integrate consumers, network platform and anchors, commodity suppliers and manufacturers, to form the complete supply end reverse supply chain. Therefore, the actors of the supply chain should focus on the focus of consumer demand, reverse through the consumer-oriented consumption and supply mode, and open through the information flow and material flow in each link. Scientific grasp of the dynamic changes of the consumption end, so that the dynamic adjustment of the supply chain behavior subjects and interconnection. All actors use shared information to newly define the three elements of "people, goods and field". They should not only take the focus of consumers, but also use scientific management means and perfect supply links to improve the quality and efficiency. Manufacturers understand the needs of reasonable preparation and customized production in advance, or the storage and preparation of relevant raw materials, in order to improve their production flexibility in response to effective orders. Logistics supply chain uses supply side and consumer order information to reasonably equip and transport, improve distribution efficiency and reduce distribution cost; platform and live broadcasting should not only understand the personalized needs of consumers, provide manufacturers with real-time consumer information, but also effectively promote new products and improve consumers' awareness and purchase desire. So as to achieve the improved quality and growth rate of the reverse supply chain.

\subsection{Strengthen Supply Chain Information Sharing Through Digital and Other Technical Means}

Under the background of the digital era, industrial digitalization has effectively promoted the production reform of enterprises, and also shortened the distance space between the supply end and the consumer end. As the subjects of actors in the live broadcasting supply chain, on the one hand, we should keep up with the trend of The Times in the macro background, embrace the arrival of the era of digital economy, and seize and obtain the dividends brought by the development of digital economy. At the micro level, we should use digital information to empower and empower enterprise production and management with upstream and downstream actors. Use digital means to improve their own information collection ability and the information sharing ability of various actors in the supply chain. On the one hand, under the premise of reasonable consumer habits data collection system, the platform establishes a perfect system, uses relevant digital tools to process and analyze the raw data, and generate the information flow needed by different actors, so that the effectiveness information is perfectly connected with the behavior subjects in each link. On the other hand, because the actors of the supply chain belong to a community with a shared future, both prosperity and damage. Behaviors of each supply chain link should also take the initiative to share information to avoid the mismatch between supply and 
demand caused by internal information blockage and affecting the common interests. Therefore, the live broadcasting platform takes advantage of the platform to reverse the effective digital information, so that the upstream enterprises can understand the needs of the consumer terminal; the upstream supply terminal through the digital information sharing, to make the anchor and the platform are targeted. All parties use the data flow to optimize the supply chain process, so as to achieve the supply situation.

\subsection{Ensure Production Stability and Improve the Service Quality of All Links of the Supply Chain}

Strictly control the raw materials of goods is the primary task of suppliers, Strengthen the supervision in the production process, The quality of the goods meets the market demand, Ensure the stable quality of each batch of the goods produced, This is conducive to reduce the frequency of return and exchange, Save off the logistics and transportation costs, Enhance customer trust; Suppliers should strengthen cooperation with live streaming e-commerce, Provide complete commodity information or physical commodity power supply provider customer service reference and understanding, Customer service of live streaming ecommerce should actively understand commodity information, Dare to face up customers' questions and answer them in details, Maintain the consistency of pre-sales and after-sales service attitude, Strengthen the service ability of after-sales customer service, After-sales customer service should clarify all the return and exchange rules and the functions of the goods, Timely reply to customers, Help customers solve problems such as how to use goods. Logistics companies should not only strengthen cooperation with manufacturers, but also strengthen contact with e-commerce platforms, clarify their responsibility relationship can reduce unnecessary disputes; improve logistics tracking technology, real-time feedback commodity transportation status and stranded reasons to the system end of e-commerce and logistics platform, facilitate customer service and consumers; future logistics process to improve service level and distribution efficiency through digital technology, including, but not limited to update product packaging style, introduce intelligent system and digital information flow to make intelligent logistics distribution. Under the effective guidance of the government regulatory departments and the continuous innovation of logistics enterprises, with the organic cooperation of the relevant actors in the future, the digital, standardization, humanization and precision of the live streaming e-commerce logistics system will be the new trend of future development.

\section{Acknowledgment}

Research project of Education Department of Liaoning Province: The impact of consumer behavior on the quality decision of agricultural supply chain 2019LNJC22.

Liaoning Provincial Social Science Planning Fund project in 2021 major project: Research on information interaction behavior of agricultural supply chain on operation quality development. L21ZD007.

\section{References}

[1] "2020 Live E-commerce White Paper" __ Live broadcast glow with vitality, driving the improvement of consumption power [J]. International Brand Watch, 2021 (08): 70-74.

[2] Shen Baogang. Analysis on the Commercial Model of Live Broadcasting with Goods and its standardized development $[\mathrm{J}]$. Theory Monthly,2020(10):59-66.

[3] Tang Siqi. Research on E-commerce Sales Mode of Live Broadcast under the Supply Chain Environment [J]. China Newspaper Industry, 2020 (06): 48-49.

[4] Li Ping. Discussion on the E-commerce Supply Chain Management Strategy in the E-commerce Era [J]. Modern marketing (Information Edition), 2020 (07): 106-107.

[5] Shen Houcai, Tao Qing, Chen Yubo. Supply Chain Management Theory and Methods [J]. China Management Science, 2000 (01): 1-9.

[6] Zhao Zizhong, Chen Lianzi. Communication theory, development status quo, industrial structure and reflection of live streaming e-commerce [J]. China Radio, 2020 (09): 11-18.

[7] Xiong Liping, Sun Ying, Fan Lanxin, Wu Xiang. Service Improvement Countermeasures of Online Purchase Supply Chain under the context of Double 11 [J]. Logistics Engineering and Management, 2021,43 (03): 92-94 + 80 .

[8] Xiong Xue, Zhu Chengxia, Zhu Haibo. The formation mechanism of consumer trust in live agricultural e-commerce: the perspective of intermediary ability [J]. Journal of Nanjing Agricultural University (Social Sciences Edition), 2021,21 (04): 142-154. 